\title{
Long Term Results of Valve Operations in Patients with Diabetes
}

\author{
Stephen J. Huddleston, Erin Goheen, Isaac Opoku-Asare, Peter Kokkinos, \\ Michael D. Greenberg, Gregory D. Trachiotis \\ Department of Surgery, Division of Cardiac Surgery, The Johns Hopkins Medical Institutions, and the Departments of Medicine \\ and Cardiology and Cardiothoracic Surgery at the Washington DC Veterans Affairs Medical Center, \\ and The George Washington University, Washington, USA \\ Email: gtrachiotis@mfa.gwu.edu
}

Received August 19, 2012; revised September 20, 2012; accepted September 30, 2012

\begin{abstract}
Diabetes Mellitus (DM) adversely affects survival in patients with Coronary Artery Disease (CAD) undergoing Coronary Artery Bypass Grafting (CABG) surgery. The influence of diabetes on events after valve operations is less defined. We analyzed the effect of diabetes on short and long term outcomes in patients undergoing valvular operations. A total of 2200 patients had cardiac surgery at a single VA Medical Center between 1991 and 2008. 355 patients had undergone valve replacement or repair. Data documenting the presence of diabetes was collected prospectively and captured into the Veterans Affairs electronic medical record. Of the 355 patients who had a valvular operation, 259 (79\%) had an Aortic Valve Replacement (AVR), 69 (20\%) had a Mitral Valve Repair/Replacement (MVR), and 4 (1\%) had a Tricsupid Valve Repair/Replacement (TVR), and 19\% $(n=69)$ of all patients had diabetes. $44 \%$ of patients with DM and $38 \%$ of patients without DM had a combined valve and CABG procedure. During a total follow up of 18 yrs, 42 (60\%) of patients with diabetes and $186(65 \%)$ of patients without diabetes were alive $(\mathrm{p}=0.118)$. At $1,5,10,15$ yrs survival in patients with versus without diabetes were 91\% v 87\%; 71\% v 74\%; 40\% v 56\%; 23\% v 48\% (p = NS). The presence of diabetes does not appear to adversely effect long-term survival in patients undergoing valve operations.
\end{abstract}

Keywords: Cardiac; Heart Valve; Diabetes

\section{Introduction}

The burden of Diabetes Mellitus (DM) in developed countries continues to increase and it is estimated that about $7 \%$ of the adult population or 20.8 million adults in the United States have DM [1,2]. It is well established that diabetics have a greater cardiovascular burden than nondiabetics, and sustain more cardiovascular events and have worse survival than non-diabetics [3,4]. Moreover, after a Myocardial Infarction (MI), diabetics have higher recurrent MI, coronary heart disease, and stroke mortality than do non-diabetics [3]. Although Coronary Artery Bypass Grafting (CABG) may attenuate the negative influence of prior MI on diabetic survival [5], overall, patients with DM that undergo CABG have increased perioperative morbidity and mortality, less freedom from recurrent angina, and significantly reduced long-term survival, even in the era of strict perioperative blood glucose control [2, 4-6].

The outcomes for diabetic patients undergoing valvular heart surgery are less well documented, and by some reports the results are conflicting [1,2,7]. Uniformly, reports suggest that patients with DM undergoing either a combined valve and CABG or isolated valve operation have increased perioperative and reduced long-term survival, yet other than the presence of diabetes, it remains unclear without a cardiovascular burden why there should be an impact on mortality. With over $30 \%$ of patients in the Society of Thoracic Surgeons national database having DM [1], it becomes important to document current outcomes for valvular heart surgery in diabetics, since type of valve operation or intervention, and choice of valve prosthesis may influence the management strategy. In this single institutional report from the Veterans Affairs Medical Center, we analyzed patients with and without DM undergoing valvular heart surgery to determine the impact of DM on hospital and long-term survival.

\section{Methods}

Between 1991-2008, 2200 patients had cardiac surgery at the Washington DC Veterans Affairs Medical Center. 362 patients were identified to either have valve replacement or repair of which complete data was available on 355. All data was captured prospectively in the Ve- 
terans Affairs electronic medical record (CPRS-VISTA), and those with and without DM were categorized and analyzed. For the entire study period, if preoperative glucose was $>250 \mathrm{mg} / \mathrm{dl}$, therapy was initiated or an insulin infusion started to achieve glucose levels $<200 \mathrm{mg} / \mathrm{dl}$, regardless of diabetic status. Postoperatively, glucose was maintained $<200 \mathrm{mg} / \mathrm{dl}$ for 24 - 48 hrs using intravenous sliding scale insulin. All diabetics were then maintained on prior standard regimen, and if glucose management was difficult to control, a specialized diabetic endocrinologist was consulted.

Diabetic patients were discharged with diabetic medication in $87 \%$ of cases. During the study period, routine use risk factor modifying medication evolved so that after 2000: antiplatelet medications were used in more than $88 \%$ of patients (initiated in 1995); statins were used in $90 \%$ (intiated in 1998); Beta-blocker usage was prevalent in 90\% (intiated in 1999); and an angiotensin converting enzyme inhibitor or angiotensin receptor blocker was used in $83 \%$ (initiated in 2000 for EF $<40 \%$ ) at the time of discharge. Patients with a bioprosthetic valve were treated with 2 months of coumadin if $<70$ yrs old in the aortic position, and all mitral or tricuspid bioprosthetic valve patients were treated for 2 months. Subsequently, all patients were maintained on ASA $81 \mathrm{mg}$ once a day. Since 2005, all patients with valvular heart diseases were enrolled and managed long-term in our combined cardiac surgery and cardiology valve clinic, and since 2007, all cardiac surgery patients with diabetes have been enrolled in a special diabetic clinic under the care of an endocrinologist specializing in cardiovascular disease.

All cause mortality was assessed utilizing both the VA Continuous Improvement in Cardiac Surgery Project (CICSP) and death data field in the VA CPRS-VISTA electronic record. Preoperative charactersitics, intraoperative variables, and postoperative complications between diabetics and non-diabetics were compared using the Student's T-test or chi-square analysis where appropriate. Long term survival curves were computed by Cox logistic regression using SPSS computer software to determine variables impacting survival. Cox proportional hazard analysis was used to control for variables that might influence survival. The Cox logistic regression and the proportional hazard analysis were performed by a statistician (PK). The authors have no financial interests, finding, or disclosures.

\section{Results}

The patient characteristics and clinical demographics are summarized in Tables 1 and 2 . The patients pre-dominantly male, and those with DM were older (67 \pm 8 versus $63 \pm 11, \mathrm{p}=0.006$ ), and had a larger BSA (2.01 ver- sus 1.96, $\mathrm{p}=0.03$ ), yet had similar rates of myocardial infarction, cardiopulmonary morbidity, prior heart surgery, and hemodynamic characteristics. The type of valve procedure, number of combined valve and CABG procedures, use of arterial graft, and bypass and cross clamp times did not differ between patients with or without DM. (Table 3). Post-operative event rates were low and similar between patients with and without DM, and operative mortality was $2.9 \%$ in DM versus $7.3 \%$ in non DM ( $p=0.18)$ and are summarized in Table 4. Long term survival beyond 18 years for combined valve and

Table 1. Patient characteristics.

\begin{tabular}{cccc}
\hline & Diabetic & Non-diabetic & p-value \\
\hline Male & $69(100 \%)$ & $\mathrm{N}=286(80.5 \%)$ & \\
Age & $67( \pm 8.8)$ & $63( \pm 11.9)$ & 0.0068 \\
BSA & $1.96( \pm 0.20)$ & $2.01( \pm 0.21)$ & 0.03 \\
Cr & $1.47( \pm 1.09)$ & $1.50(1.06)$ & 0.86 \\
\hline
\end{tabular}

Table 2. Preoperative risk factors and comorbidities.

\begin{tabular}{lccc}
\hline & Diabetic & Non-diabetic & p-value \\
\hline Cardiomyopathy & $36(52.2 \%)$ & $168(58.7 \%)$ & 0.32 \\
COPD & $36(52.2 \%)$ & $123(43 \%)$ & 0.17 \\
Prior heart surgery & $7(10.1 \%)$ & $25(8.7 \%)$ & 0.72 \\
Prior MI & $22(31.9 \%)$ & $72(25 \%)$ & 0.28 \\
Preop IABP & $4(5.8 \%)$ & $13(4.5 \%)$ & 0.66 \\
PVD & $25(36.2 \%)$ & $69(24 \%)$ & 0.041 \\
LVEDP (mmHg) & $20.0( \pm 11.8)$ & $20.4( \pm 10.1)$ & 0.81 \\
Mean PAP (mmHg) & $18.96( \pm 8.58)$ & $18.0( \pm 8.7)$ & 0.49 \\
\hline
\end{tabular}

Table 3. Operative data.

\begin{tabular}{lccc}
\hline & Diabetic & Non-diabetic & p-value \\
\hline AVR & $55(79.7 \%)$ & $204(71.3 \%)$ & 0.16 \\
MVR & $11(15.9 \%)$ & $58(20.3 \%)$ & 0.42 \\
TVR & $1(1.4 \%)$ & $3(1 \%)$ & 0.78 \\
Repair & $4(5.8 \%)$ & $25(8.74 \%)$ & 0.42 \\
CABG + Valve & $31(44.9 \%)$ & $105(36.7 \%)$ & 0.35 \\
Arterial graft & $19(27.5 \%)$ & $52(18.2 \%)$ & 0.087 \\
CPB time & $117( \pm 35)$ & $133( \pm 61)$ & 0.082 \\
Ischemic time & $81( \pm 25)$ & $89( \pm 30)$ & 0.113 \\
\hline
\end{tabular}


CABG (Figure 1(a)) and isolated valve surgery (Figure 1(b)) were similar between patients with or without DM $(p=N S)$. There was also no difference in long term survival between diabetics who underwent combined valve and CABG versus isolated valve surgery (data not shown). Cox regression analysis for influence of survival for the study population demonstrated that prior heart surgery $(\mathrm{HR}=1.94$; CI 95\% = $1.14-3.31)$, renal failure $(\mathrm{HR}=$ 2.14; CI $95 \%=3.04-16.78$ ) and a history of cerebrovascular disease $(\mathrm{HR}=1.7$; CI 95\% $=1.2-2.57)$ influenced survival. Other variables that did not influence survival included diabetes, a history of COPD or prior MI, cigarette smoking, previous cerebrovascular accident, or perioperative MI (Table 5).

\section{Discussion}

The number of patients with diabetes presenting for cardiac surgery continues to increase, and accounts for $>30 \%$ of patients in the STS database [1,4,7] Diabetes remains a major risk factor for morbidity and mortality after CABG, even in the era of tight glucose control. [1, 3-5] The influence of diabetes on survival after valve surgery is unclear, and has not been defined in a study that has maintained rigid perioperative glucose control or instituted long-term risk factor modifying therapy $[2,7]$. The influence of diabetes becomes important when presenting these patients with the options for valve therapy $[8,9]$. We examined a prospectively maintained database of cardiac surgery including 362 valve operations over 17 years at a single VA medical center to determine the influence of diabetes on hospital and long-term outcome after valve operations. Complete data on 355 patients and long -term survival was determined using the EMR and another quality improvement database. While renal failure, cerebrovascular disease and prior heart surgery negatively impacted long-term survival, diabetes mellitus had no statistically significant impact on hospital or longterm survival. The similar survival between diabetics and non-diabetics undergoing valve surgery may be due to similar coronary artery disease burden between these two particular groups of patients, and lack of extensive Left

Table 4. Complications and operative mortality.

\begin{tabular}{lccc}
\hline & Diabetic & Non-diabetic & p-value \\
\hline Periop. MI & $1(1.45 \%)$ & $7(2.45 \%)$ & 0.62 \\
Renal failure & $2(2.9 \%)$ & $6(2.1 \%)$ & 0.69 \\
Reop. Bleed & $3(4.3 \%)$ & $13(4.5 \%)$ & 0.94 \\
Vent > 48 hrs & $7(10.1 \%)$ & $26(9.1 \%)$ & 0.79 \\
Stroke & $1(1.4 \%)$ & $6(2.1 \%)$ & 0.78 \\
Any complication & $10(14.5 \%)$ & $60(21 \%)$ & 0.23 \\
30-day mortality & $2(2.9 \%)$ & $21(7.3 \%)$ & 0.18 \\
\hline
\end{tabular}

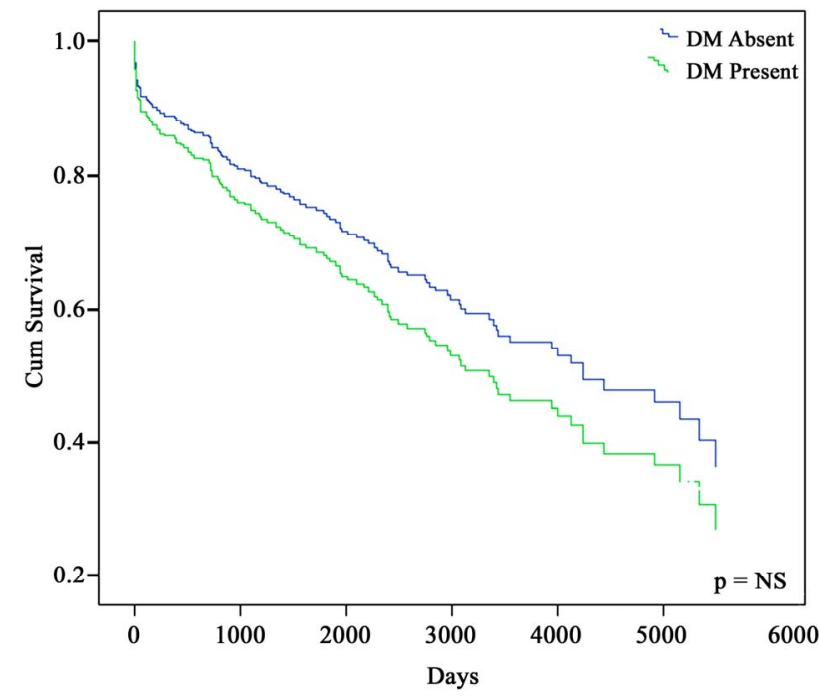

(a)

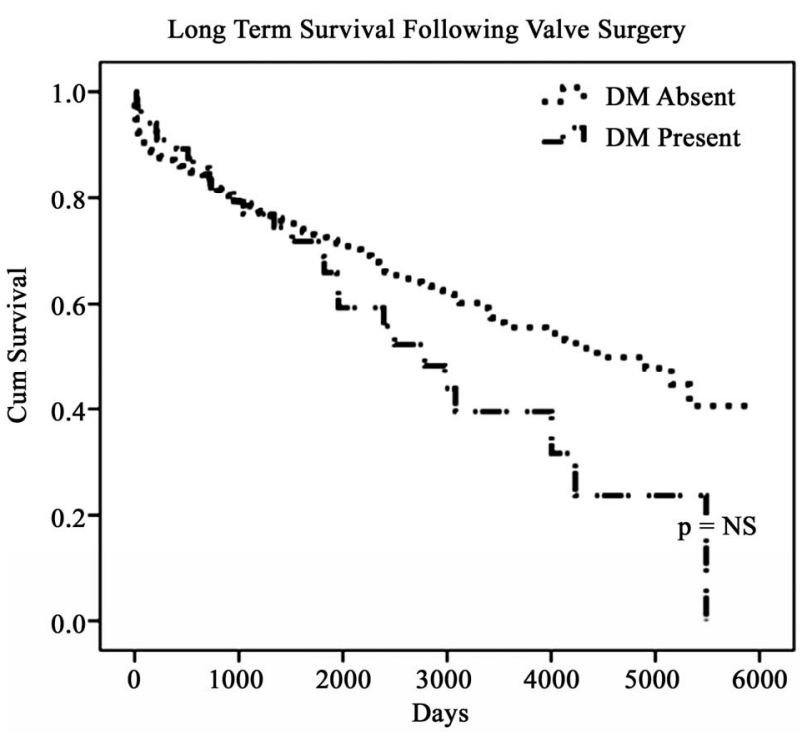

(b)

Figure 1. (a) Survival in diabetic versus non-diabetic patients after all valve surgery; (b) Survival in diabetics versus nondiabetics after isolated valve surgery.

Table 5. Risk factors influencing survival.

\begin{tabular}{lccc}
\hline \multicolumn{1}{c}{ Variable } & N (\%) & Hazard ratio (RR) & CI (95\%) \\
\hline Renal failure & 8 & 7.14 & $3.04-16.78$ \\
CVD & 83 & 1.76 & $1.20-2.57$ \\
Prior heart surgery & 4 & 1.94 & $1.14-3.31$ \\
\hline
\end{tabular}

Anterior Descending artery (LAD) disease in the patients (only about $18 \%$ - $27 \%$ to the LAD distribution as demonstrated by IMA usage). Since grafting of the left anterior descending artery with the left internal mammary artery is the primary determinant of improved long term survival after CABG $[6,10]$ and utilized in $99 \%$ of our 
practice, the lack of difference in this variable between the two groups could explain the similar survival between combined valve and CABG versus isolated valve patients with or without diabetes, since an predictive burden of cardiovascular disease does not exist. [2,5,7] Also, in diabetics undergoing isolated CABG, pulmonary and systemic hypertension are significantly more prevalent compared to non-diabetics, and this distinction does not exist for these groups undergoing valvular heart surgery [5]. It is interesting to note, that in diabetics undergoing valve operations a prior myocardial infarction did not influence survival, a feature we also found in diabetic undergoing isolated CABG [5]. Therefore, the impact of remodeling or structural cardiac changes influenced by microvascular disease may not exist in diabetics with primarily valvular heart disease [1,2-6].

One important feature of our study is that in-hospital and perioperative morbiditiy and mortality is no different between patients with DM or without DM. This is in distinction from other reports on valve heart surgery in diabetics $[1,2,7]$ This difference may be attributed to our postoperative management strategy that included a strict regimen of perioperative glucose control $<200 \mathrm{mg} / \mathrm{dl}$ [5]. Although the influence of perioperative glucose control has been a subject of debate or controversy, for diabetics undergoing $\mathrm{CABG}$, it has shown to improve perioperative outcomes $[1,4,5]$. Therefore, tight perioperative glucose control and implementation of other risk factor modifying therapies in this study may have favorably impacted outcomes. For all patients having valve heart surgery (with or without CABG), there is no significant long-term survival difference between diabetics or nondiabetics. Nonetheless, a trend toward decreased survival beyond 15 years can be seen among diabetic patients after valve surgery compared to non-diabetic patients. It is possible that the lack of statistical difference between the two groups is due to the small sample size in this study or perhaps due to the increased LAD territory grafted in diabetic undergoing combined valve and CABG versus nondiabetics $(27.5 \% \mathrm{v} 18.2 \%, \mathrm{p}=0.087)$. For the entire study population, renal failure and cerebrovascular disease worsened survival. Since the prevalence of these risk factors is greater among diabetics, perhaps the impact on survival is not seen until much later. On the other hand, the implementation of risk factor modifying therapy, and a cohesive and coordinated follow-up strategy with a valve and endocrine clinic may have attenuated the diabetic disease burden on long-term survival. Since long term survival beyond 15 years is excellent for diabetics undergoing isolated valve or valve and CABG to non-LAD territories, a decision on techniques of valve therapy or choice of valve should not be premised on diabetes alone. With emerging less invasive valve techniques, such as percutaneous mitral repair or aortic valve replacement (TAVI), where decision factors other than age may become important, it remains critical to clarify influence of survival. Moreover, the choice of valve prosthesis is not only influenced by age of patient, and a preference to avoid coumadin, but also coexistence of extensive CAD and cormobidities [8]. Based on our review, diabetes should not be a factor influencing choice of valve prosthesis. For example, in an otherwise healthy diabetic undergoing aortic or mitral valve surgery with our without CABG to non LAD territory who is in their 60's, it would be acceptable practice to put in a bioprosthetic valve. On the other hand in a functional $80+$ year old with aortic valve disease with diabetes, it is absolutely the standard to perform conventional aortic valve replacement versus transluminal aortic valve insertion (TAVI).

\section{Conclusion}

In summary, patients with diabetes undergoing isolated or combined valve and CABG have excellent perioperative outcomes and long-term survival compared to a non diabetic population. Postoperative and long term glucose management strategies, and risk factor modifying therapies, including enrollment into valve clinic and diabetic teaching clinics remain important strategies for improving survival. The presence of diabetes itself should not impact the valve therapy or choice of valve prosthesis.

\section{REFERENCES}

[1] J. R. Brown, F. H. Edwards, G. T. O’Conner, C. S. Ross and A. P. Furnary, “The Diabetic Disadvantage: Historical Outcomes Measures in Diabetic Patients Undergoing Cardiac Surgery-The Pre-Intravenous Insulin Era," Seminars in Thoracic and Cardiovascular Surgery, Vol. 18, No. 4, 2006, pp. 281-288. doi:10.1053/j.semtcvs.2006.04.004

[2] S. Castelvecchio, L. Menicanti, E. Baryshnikova, C. de Vincentiis, A. Frigiola and M. Ranucci, "For the Surgical and Clinical Outcome Research (SCORE) Group. Comparison of Morbidity and Mortality in Diabetics versus Nondiabetics Having Isolated Coronary Artery Bypass versus Coronary Bypass Plus Valve Operations versus Isolated Valve Operations,” American Journal of Cardiology, Vol. 107, No. 4, 2011, pp. 535-539. doi:10.1016/j.amjcard.2010.10.009

[3] C. D. Lee, A. R. Folsom, J. S. Pankow and F. L. Brancati, "For the Atherosclerosis Risk in Communities (ARIC) Study Investigators. Cardiovascular Events in Diabetic and Nondiabetic Adults with or without History of Myocardial Infarction,” Circulation, Vol. 109, 2004, pp. 855-860. doi:10.1161/01.CIR.0000116389.61864.DE

[4] H. L. Lazar, S. R. Chipkin, C. A. Fitzgerald, Y. Bao, H. Cabral and C. S. Apstein, "Tight Glycemic Control in Diabetic Coronary Artery Bypass Graft Patients Improves Perioperative Outcomes Ans Decreases Recurrent Ischemic Events," Circulation, Vol. 109, 2004, pp. 1497-1502. doi:10.1161/01.CIR.0000121747.71054.79 
[5] G. Trachiotis, P. Kokkinos and M. Greenberg, "LongTerm Survival after CABG in Diabetics with Aggressive Risk Factor Management," Interactive Cardiovascular Thoracic Surgery, Vol. 7, 2008, pp. S2-S3.

[6] G. D. Trachiotis, W. S. Weintraub, T. S. Johnston, E. L. Jones, R. A. Guyton and J. M. Craver, "Coronary Artery Bypass Grafting in Patients with Advanced Left Ventricular Dysfunction,” The Annals of Thoracic Surgery, Vol. 66, 1998, pp. 1632-1639. doi:10.1016/S0003-4975(98)00773-5

[7] M. E. Halkos, P. Kilgo, O. M. Lattouf, J. D. Puskas, W. A. Cooper, R. A. Guyton and V. H. Thourani, "The Effect of Diabetes Mellitus on in-Hospital and Long-Term outcomes after Heart Valve Operations," The Annals of Thoracic Surgery, Vol. 90, 2010, pp. 124-130.

doi:10.1016/j.athoracsur.2010.03.111
[8] S. H. Rahimtoola, "Choice of Prosthetic Heart Valve in Adults an Update," Journal of the American College of Cardiology, Vol. 55, No. 22, 2010, pp. 2413-2426. doi:10.1016/j.jacc.2009.10.085

[9] T. E. David, S. Armstrong and Z. Sun, "Left Ventricular Function after Mitral Valve Surgery,” Journal of Heart Valve Disease, Vol. 4, No. 2, 1995, pp. 175-180.

[10] R. H. Zeff, C. Kongtahworn, L. A. Iannone, D. F. Gordon, T. M. Brown, S. J. Phillips, J. R. Skinner and M. Spector, "Internal Mammary Artery versus Saphenous Vein Graft to the Left Anterior Descending Coronary Artery: Prospective Randomized Study with 10-Year Follow-up," The Annals of Thoracic Surgery, Vol. 45, No. 5, 1988, pp. 533-536. doi:10.1016/S0003-4975(10)64526-2 